\title{
Serum HBV RNA Predicts HBeAg Clearance and Seroconversion in Patients with Chronic Hepatitis B Treated with Nucleos(t)Ide Analogues
}

\section{Yang Wang}

Capital Medical University Youan Hospital

Hao Liao

Peking University Health Science Centre

\section{Zhongping Deng}

Peking University Academy for Advanced Interdisciplinary Studies

Yanna liu

Peking University Health Science Centre

\section{Dandan bian}

Capital Medical University Youan Hospital

\section{Yan Ren}

Capital Medical University Youan Hospital

\section{Guangxin Yu}

Peking University Health Science Centre

\section{yingying Jiang}

Capital Medical University Youan Hospital

\section{Li Bai}

Capital Medical University Youan Hospital

\section{Shuang Liu}

Capital Medical University Youan Hospital

Mei Liu

Capital Medical University Youan Hospital

\section{Li Zhou}

Capital Medical University Youan Hospital

\section{Yu Chen}

Capital Medical University Youan Hospital

\section{Zhongping Duan}

Capital Medical University Youan Hospital

\section{Sujun Zheng ( $\nabla$ zhengsujun@ccmu.edu.cn )}

Capital Medical University Youan Hospital https://orcid.org/0000-0002-6367-5764

fengmin Lu 


\section{Research Article}

Keywords: Chronic hepatitis B, HBV DNA, HBV RNA, HBcrAg, HBsAg, intrahepatic HBV DNA, cccDNA, $\mathrm{HBeAg}$ clearance, $\mathrm{HBeAg}$ seroconversion, nucleos(t)ide analogues

Posted Date: July 16th, 2021

DOl: https://doi.org/10.21203/rs.3.rs-707823/v1

License: (c) (i) This work is licensed under a Creative Commons Attribution 4.0 International License. Read Full License

Version of Record: A version of this preprint was published at Journal of Viral Hepatitis on March 10th, 2022. See the published version at https://doi.org/10.1111/jvh.13671. 


\section{Abstract}

Background: To evaluate the predictive value of serum HBV DNA, HBV RNA, HBcrAg, HBsAg, intrahepatic HBV DNA, and cccDNA for HBeAg clearance and seroconversion during long-term treatment of nucleos(t)ide analogues (NAs) in patients with chronic hepatitis $B(C H B)$.

Method: A single center, prospective cohort of CHB patients enrolled between June 2007 and July 2008 was used for this study. Serum HBV RNA levels were retrospectively measured at baseline, 6, 12, 24, 36, $48,60,72$, and 84 months post-NAs treatment. Serum HBsAg and HBcrAg levels were quantified at baseline, month 6,60 , and 72 . Histological sample from liver biopsy at baseline and month 60 were analyzed for intrahepatic HBV DNA and cccDNA.

Results: Eighty-three HBeAg patients were enrolled with an median follow-up time of 108 months (range 18-138 months). Of them, 53 (63.86\%) patients achieved HBeAg clearance, and 37 (44.58\%) achieved HBeAg seroconversion. Only baseline HBV RNA was independently associated with HBeAg clearance (OR=0.50, 95\% $\mathrm{Cl} 0.309-0.809, P=0.005$ ) and seroconversion ( $\mathrm{OR}=0.689,95 \% \mathrm{Cl} 0.513-0.925, P=0.013$ ). The independent negative association with $\mathrm{HBeAg}$ clearance and seroconversion remained for HBV RNA levels at month $6(\mathrm{OR}=0.42,95 \% \mathrm{Cl} 0.248-0.714, P=0.001 ; \mathrm{OR}=0.44,95 \% \mathrm{Cl} 0.260-0.744, P=0.002)$ and month 12 (OR=0.39, 95\% $\mathrm{Cl} 0.253-0.592, P<0.001 ; \mathrm{OR}=0.58,95 \% \mathrm{Cl} 0.427-0.798, P=0.001)$. The AUC of baseline HBV RNA for predicting the HBeAg clearance and seroconversion were 0.81 ( $95 \% \mathrm{Cl}$ : 0.70-0.89) and 0.68 (95\% Cl: $0.56-0.78)$, respectively, higher than that of HBV DNA, HBsAg and HBcrAg.

Conclusion: Lower serum HBV RNA at baseline, month 6 and 12 post NAs treatment could predict HBeAg clearance and seroconversion during long-term NAs treatment.

\section{Introduction}

Hepatitis B virus (HBV) infection has been a global public health challenge [1]. Nucleos(t)ide analogues (NAs), which suppresses HBV DNA replication via inhibiting the reverse transcription of pregenomic RNA (pgRNA) into HBV DNA, are considered the first-line treatment option. However, NAs fails to clear the HBV covalently closed circular DNA (cccDNA) and RNA replicative intermediates [2]. Thus, long-term and even indefinite NAs treatment course is needed for sustained viral suppression in chronic hepatitis $B(C H B)$ patients. The ideal anti-HBV treatment endpoint, namely functional cure is serum hepatitis $B$ surface antigen (HBsAg) clearance [2]. For hepatitis $\mathrm{B}$ e antigen (HBeAg) positive CHB patients, the realistic goal is to attain HBeAg clearance and further achieve seroconversion, which reflect partial immune control and pre-requisite for the ideal treatment endpoint [2]. Therefore, biomarkers are urgently needed to predict the possibility of $\mathrm{HBeAg}$ response during long-term NAs treatment.

Viral markers including serum HBV DNA, HBV RNA, hepatitis B core-related antigen (HBcrAg) and quantitative HBsAg are currently used to predict therapeutic response for $\mathrm{CHB}$ patients undergoing NAs therapy $[3,4]$. It has been reported that on-treatment decline of HBsAg level at 48 week or 96 week may help predict the HBeAg clearance or seroconversion in HBeAg positive CHB patients treated with NAs [5, 
6]. Besides, HBV DNA levels at month 6 and 12 could predict serum HBeAg clearance in this population treated with Entecavir [7, 8]. However, the predictability of HBsAg in Lee's study [9] and HBV DNA in Shin's study [10] for HBeAg seroconversion cannot be repeated in Fung's study [11] in HBeAg positive CHB patients treated with NAs.

Serum HBV RNA is the encapsidated HBV pregenomic RNA (pgRNA) existed inside the virion-like particles [12]. It reflects the intrahepatic transcriptional activity of cccDNA and indicates changes of liver inflammation and fibrosis score in CHB patients receiving NAs therapy [13]. Luo et al. reported that HBeAg seroconversion occurred in CHB patients with HBV RNA levels below $4.12 \log _{10}$ copies $/ \mathrm{mL}$ before NAs treatment [14]. And patients who remained HBV RNA positive after 48 weeks of NAs treatment had an increased risk of not achieving HBeAg clearance [15]. Whether HBV RNA can predict both HBeAg clearance and seroconversion during long-term NAs treatment remains unknown.

$\mathrm{HBcrAg}$ is also considered a novel serum marker for disease monitoring and prognosis of CHB [16]. Compared to serum HBsAg and HBV DNA level, serum HBcrAg had a better correlation with intrahepatic cccDNA [17]. And evidence is accumulating that HBcrAg could predict the response to NAs therapy, offtreatment viral relapse, HBeAg seroconversion, and the risk of development to HCC [18]. Wang and colleagues revealed that the quantitative serum HBsAg and HBcrAg could predict the HBeAg seroconversion in HBeAg positive patients treated with NAs [19].

In this single center, longitudinal study, we evaluate the predictive value of serum viral markers including HBV DNA, HBV RNA, HBcrAg, HBsAg, intrahepatic HBV DNA and liver tissue cccDNA for HBeAg clearance and seroconversion during long-term treatment with NAs in HBeAg-positive CHB patients.

\section{Material And Methods}

\section{Patients and study design}

This study has been conducted using the database of patients receiving NAs monotherapy, prospectively recruited from Beijing YouAn Hospital, Capital Medical University (Beijing, China) between June 2007 and July 2008. Eligible patients were diagnosed CHB according to the AASLD [20], male or female patients aged $\geq 16$ years. The exclusion criteria were as follows: i) co-infection with another viruses, including hepatitis $C$ or $D$ virus, Epstein-Barr virus, cytomegalovirus and human immunodeficiency viruses, the existence of autoimmune liver disease, or alcoholic liver disease; ii) with decompensated liver function (ascites, hepatic encephalopathy or upper gastrointestinal bleeding); iii) with any diseases of other major organs, such as severe heart disease or kidney disease; iv) poor compliance; v) history of a malignancy, including hepatocellular carcinoma, carcinoma in situ and atypical hyperplastic nodules; vi) with mental illness; vii) had received corticosteroids, immunosuppressants or chemotherapeutic drugs $\leq 6$ months prior to enrollment; and viii) pregnant or breast-feeding women. 
Patients were followed-up every three months in the first year of therapy, and every six months thereafter. At each follow-up, serum specimens were collected for liver function tests and HBV DNA quantification. Serum HBsAg, anti-HBs, HBeAg, anti-HBe, and anti-HBc were also determined. Remaining serum samples were stored at $-80^{\circ} \mathrm{C}$ for subsequent research. At enrollment and 60 months on-treatment, percutaneous liver biopsy was performed to evaluate the histological changes, which were diagnosed according to the modified Knodell and Ishak scoring system [21].

$\mathrm{HBeAg}$ clearance was defined as the cut-off index (COI) of HBeAg lower than 1. HBeAg seroconversion was defined as the $\mathrm{COI}$ of HBeAg lower than 1 and the $\mathrm{COI}$ of anti-HBe was greater than 1 .

With the foregoing collected blood, we retrospectively quantified HBsAg and HBcrAg levels at baseline, 6 , 60 and 72 months, HBV RNA levels at baseline, 6, 12, 24, 36, 48, 60, 72 and 84 months, and intrahepatic HBV DNA and cccDNA levels at baseline and at 60 months by a method previously reported [3, 12, 22].

The study was conducted in compliance with the Declaration of Helsinki. Use of the research samples was approved by the Medical Ethics Review Committee of Beijing YouAn Hospital. All patients provided written informed consent authorizing us to access their medical records and to store the serum specimens for research purposes.

\section{Assays for serological HBV markers, HBV DNA, HBV RNA, and HBcrAg}

Serum HBsAg, anti-HBs, HBeAg, anti-HBe, and anti-HBc were determined on a Roche Cobas e601 analyzer using an electrochemiluminescence immunoassay (Abbott Laboratories, Chicago, IL, USA). HBsAg was quantified using an Elecsys for HBsAg quantitation (Roche Diagnostics) with a lower limit of detection (LLD) of $0.05 \mathrm{IU} / \mathrm{mL}$. The serum HBV DNA level was determined using the Cobas HBV Amplicor Monitor assay (Roche Diagnostics, Pleasanton, CA, USA), with a LLD of $50 \mathrm{IU} / \mathrm{mL}$. Details for HBV RNA assay could be found in Supplementary Materials. HBcrAg was determined using chemiluminescent enzyme immunoassay in automated analyzer system (Lumipulse System, Fujirebio Inc., Tokyo, Japan). The LLD was $1,000 \mathrm{U} / \mathrm{mL}$ with a linear range of $3-7 \log _{10} \mathrm{U} / \mathrm{mL}$.

\section{Quantitation of intrahepatic HBV DNA and cccDNA}

The DNA was extracted from FFPE liver biopsy tissue using QIAamp FFPE DNA Mini Kit (QIAGEN, GmbH, Hilden, Germany) according to the instructions of the manufacturer. Details could be found in Supplementary Materials.

\section{Statistical analysis}

HBsAg, HBV DNA, HBV RNA, HBcrAg, intrahepatic HBV DNA and cccDNA expression were logarithmically transformed for analysis. Continuous variables were expressed as mean \pm SD or median (range). Categorical data were expressed as counting and/or proportion. Continuous quantitative data were compared between groups using $t$ test or Mann-Whitney- $U$ test, the comparison between before and after treatment was performed using Wilcox rank sum test. The comparison between the counting data sets 
was performed using a chi-square test or Fisher's exact probabilities. Logistic multivariate regression analysis was used to determine relevant factors independently associated with HBeAg clearance and seroconversion, using moving forward method, $P$ values of entry and removal were 0.05 and 0.10 , respectively. In Kaplan-Meier survival analysis, patients were divided into two specified groups based on median value of continuous variables, in the aim to compare the accumulated HBeAg clearance and seroconversion rate of specified groups. Patients who were lost to follow-up before HBeAg clearance or seroconversion were considered as HBeAg non-clearance or non-seroconversion. The predictive capacity of these serum biomarkers were assessed by the receiver operating characteristic (ROC) analysis with the cut-off values determined using Youden index. Comparisons between ROC curves were performed using DeLong's test. Based on the predictive probability of logistic multivariate regression analysis, various combinations of HBsAg, HBV DNA, HBV RNA and HBcrAg were evaluated to predict HBeAg clearance and seroconversion at baseline, at month 6 and 12. Data were analyzed using the IBM SPSS 22.0 (SPSS Inc., Chicago, IL, USA). $P<0.05$ was considered statistically significant in two-tailed test.

\section{Results}

\section{Patients}

A total of 83 eligible $\mathrm{HBeAg}$ positive $\mathrm{CHB}$ patients were enrolled. Of them, 36 were NAs-naïve patients, 47 were Lamivudine treated patients. Forty-six (55.42\%) patients were treated with Entacavir $0.5 \mathrm{mg}$ once daily and the other 37 (44.58\%) patients were treated with Adefovir dipivoxil 10mg once daily. Sixty-eight (81.93\%) patients were male. Average age was $36.42 \pm 9.78$ years. The median follow-up period was 108 months (range: 18-138 months). Thirty-nine patients with available genotype data, with $74.36 \%(29 / 39)$ patients were genotype $\mathrm{C}$. Fifty-nine patients obtained baseline liver biopsy, the median inflammation and fibrosis score were 7 (range: 2-15) and 3 (range: 1-5), respectively. Detailed demographic and clinical characteristics were shown in Table 1. 
Table 1

Baseline characteristics and clinical variables related to $\mathrm{HBeAg}$ clearance by univariate analyses

$\begin{array}{lllll}\text { Variables } & \text { Total } & \begin{array}{l}\text { HBeAg } \\ \text { clearance } \\ (n=83)\end{array} & \begin{array}{l}\text { HBeAg non- } \\ \text { clearance } \\ (n=53)\end{array} & \begin{array}{l}P \\ \text { valuet }\end{array} \\ & & (n=30) & \end{array}$

\section{Baseline characteristics}

\begin{tabular}{lllll} 
Age, years & $36.42 \pm$ & $38.32 \pm 10.43$ & $33.07 \pm 7.58$ & 0.010 \\
\hline Male (\%) & 9.78 & & & \\
\hline ETV/ADV & $68(81.93)$ & $42(79.25)$ & $26(86.67)$ & 0.399 \\
\hline BMI kg/m ${ }^{2}$ & $46 / 37$ & $26 / 27$ & $20 / 10$ & 1.121 \\
\hline & $23.89 \pm$ & $23.81 \pm 3.91$ & $24.02 \pm 3.40$ & 0.807 \\
\hline
\end{tabular}

HBV Genotype ††

\begin{tabular}{lllll} 
B/C/others & $5 / 29 / 5$ & $2 / 19 / 2$ & $3 / 10 / 3$ & 0.368 \\
ALT, U/L & $98.37 \pm$ & $103.38 \pm$ & $89.53 \pm 83.62$ & 0.573 \\
& 106.73 & 118.29 & & \\
\hline AST, U/L & $59.49 \pm$ & $65.68 \pm 60.23$ & $48.56 \pm 37.45$ & 0.213 \\
\hline & 53.52 & & &
\end{tabular}

AST/ALT ratio

$0.76 \pm 0.34$

$0.77 \pm 0.31$

$0.72 \pm 0.39$

0.240

TBIL, $\mu \mathrm{mol} / \mathrm{L}$

$16.68 \pm$

$17.85 \pm 9.18$

$14.91 \pm 4.27$

0.381

7.65

ALP, U/L

$105.00 \pm$

60.06

$109.28 \pm$

56.27

$\mathrm{HBsAg}\left(\log _{10} \mathrm{IU} / \mathrm{mL}\right)$

$3.60 \pm 1.04$

$3.28 \pm 0.87$

$3.83 \pm 1.26$

0.001

HBV DNA $\left(\log _{10} \mathrm{IU} / \mathrm{mL}\right)$

$6.44 \pm 1.95$

$5.93 \pm 1.99$

$7.36 \pm 1.53$

0.001

HBV RNA ( $\log _{10}$ copies $/ \mathrm{mL}$ )

$5.38 \pm 1.71$

$4.38 \pm 1.56$

$6.22 \pm 1.41$

0.000

Table data are number of patients or median (range), or mean \pm standard deviation.

tP values are acquired by univariate analyses between $\mathrm{HBeAg}$ clearance and non-clearance..

†† Thirty-nine patients with available genotype data were analyzed.

㧊 Fifty-nine patients with available liver biopsy data were analyzed. Hepatic inflammation grade and fibrosis stage were diagnosed according to the modified Knodell and Ishak scoring system respectively.

Abbrevation: ADV, Adefovir; ALP, alkaline phosphatase; ALT, alanine aminotransferase; AST, aspartate aminotransferase; BMI, body mass index; cccDNA, covalently closed circular DNA; ETV, Entecavir; HBV, hepatitis B virus; HBV RNA, hepatitis B virus ribonucleic acid; $\mathrm{HBcrAg}$, hepatitis B core-related antigen; HBeAg, hepatitis B e antigen; HBsAg, hepatitis B surface antigen; TBIL, total bilirubin. 


\begin{tabular}{|c|c|c|c|c|}
\hline Variables & $\begin{array}{l}\text { Total } \\
(n=83)\end{array}$ & $\begin{array}{l}\mathrm{HBeAg} \\
\text { clearance } \\
(n=53)\end{array}$ & $\begin{array}{l}\text { HBeAg non- } \\
\text { clearance } \\
(n=30)\end{array}$ & $\begin{array}{l}P \\
\text { valuet }\end{array}$ \\
\hline $\mathrm{HBcrAg}\left(\log _{10} \mathrm{U} / \mathrm{mL}\right)$ & $7.15 \pm 1.12$ & $6.64 \pm 1.12$ & $7.74 \pm 0.74$ & 0.000 \\
\hline $\begin{array}{l}\text { Intrahepatic HBV DNA } \\
\left(\log _{10} \text { copies } / 10^{5} \text { cell) }\right.\end{array}$ & $6.37 \pm 0.83$ & $6.21 \pm 0.76$ & $6.59 \pm 0.90$ & 0.101 \\
\hline $\operatorname{cccDNA}\left(\log _{10}\right.$ copies $/ 10^{5}$ cell) & $4.74 \pm 0.93$ & $4.45 \pm 0.76$ & $5.10 \pm 1.04$ & 0.116 \\
\hline Inflammation scores㧊 & $8.58 \pm 3.84$ & $9.64 \pm 4.15$ & $6.93 \pm 2.62$ & 0.018 \\
\hline 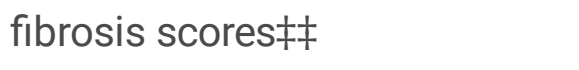 & $3.07 \pm 0.95$ & $3.32 \pm 0.84$ & $2.67 \pm 1.00$ & 0.007 \\
\hline \multicolumn{5}{|l|}{ At 6 month time-point } \\
\hline ALT, U/L & $\begin{array}{l}39.06 \pm \\
30.90\end{array}$ & $37.56 \pm 25.57$ & $41.74 \pm 39.05$ & 0.747 \\
\hline AST, U/L & $\begin{array}{l}31.37 \pm \\
14.48\end{array}$ & $32.30 \pm 15.18$ & $29.72 \pm 13.26$ & 0.493 \\
\hline AST/ALT ratio & $0.95 \pm 0.30$ & $0.98 \pm 0.29$ & $0.89 \pm 0.30$ & 0.238 \\
\hline TBIL, $\mu \mathrm{mol} / \mathrm{L}$ & $\begin{array}{l}14.58 \pm \\
6.75\end{array}$ & $17.35 \pm 8.06$ & $17.42 \pm 4.76$ & 0.892 \\
\hline ALP, U/L & $\begin{array}{l}97.50 \pm \\
31.83\end{array}$ & $\begin{array}{l}102.75 \pm \\
32.57\end{array}$ & $81.08 \pm 24.23$ & 0.044 \\
\hline HBsAg & $3.54 \pm 0.84$ & $3.45 \pm 0.64$ & $3.66 \pm 1.05$ & 0.074 \\
\hline HBV DNA (log10 IU/mL ) & $3.11 \pm 1.36$ & $2.71 \pm 1.24$ & $3.79 \pm 1.32$ & 0.000 \\
\hline HBV RNA (log10 copies/mL ) & $4.53 \pm 1.83$ & $3.65 \pm 1.55$ & $5.93 \pm 1.31$ & 0.000 \\
\hline HBcrAg & $6.64 \pm 1.10$ & $6.07 \pm 0.92$ & $7.37 \pm 0.86$ & 0.000 \\
\hline
\end{tabular}

Table data are number of patients or median (range), or mean \pm standard deviation.

tP values are acquired by univariate analyses between $\mathrm{HBeAg}$ clearance and non-clearance..

t† Thirty-nine patients with available genotype data were analyzed.

㧊 Fifty-nine patients with available liver biopsy data were analyzed. Hepatic inflammation grade and fibrosis stage were diagnosed according to the modified Knodell and Ishak scoring system respectively.

Abbrevation: ADV, Adefovir; ALP, alkaline phosphatase; ALT, alanine aminotransferase; AST, aspartate aminotransferase; BMI, body mass index; cccDNA, covalently closed circular DNA; ETV, Entecavir; HBV, hepatitis B virus; HBV RNA, hepatitis B virus ribonucleic acid; HBcrAg, hepatitis B core-related antigen; HBeAg, hepatitis B e antigen; HBsAg, hepatitis B surface antigen; TBIL, total bilirubin. 


\begin{tabular}{|c|c|c|c|c|}
\hline Variables & $\begin{array}{l}\text { Total } \\
(n=83)\end{array}$ & $\begin{array}{l}\mathrm{HBeAg} \\
\text { clearance } \\
(\mathrm{n}=53)\end{array}$ & $\begin{array}{l}\text { HBeAg non- } \\
\text { clearance } \\
(n=30)\end{array}$ & $\begin{array}{l}P \\
\text { valuet }\end{array}$ \\
\hline$A L T, U / L$ & $\begin{array}{l}31.97 \pm \\
21.43\end{array}$ & $33.07 \pm 23.62$ & $30.07 \pm 17.23$ & 0.614 \\
\hline AST, U/L & $\begin{array}{l}28.30 \pm \\
11.37\end{array}$ & $29.51 \pm 11.81$ & $26.21 \pm 10.45$ & 0.149 \\
\hline AST/ALT ratio & $1.06 \pm 0.44$ & $1.09 \pm 0.49$ & $1.00 \pm 0.35$ & 0.368 \\
\hline TBIL, $\mu \mathrm{mol} / \mathrm{L}$ & $\begin{array}{l}14.70 \pm \\
7.18\end{array}$ & $16.74 \pm 8.12$ & $17.61 \pm 8.03$ & 0.865 \\
\hline ALP, U/L & $\begin{array}{l}94.55 \pm \\
50.65\end{array}$ & $\begin{array}{l}100.99 \pm \\
55.29\end{array}$ & $78.91 \pm 33.79$ & 0.129 \\
\hline HBV DNA $\left(\log _{10} \mathrm{IU} / \mathrm{mL}\right)$ & $2.83 \pm 1.51$ & $2.46 \pm 1.27$ & $3.49 \pm 1.68$ & 0.001 \\
\hline HBV RNA ( $\log _{10}$ copies/mL ) & $4.18 \pm 1.87$ & $3.28 \pm 1.45$ & $5.63 \pm 1.54$ & 0.000 \\
\hline \multicolumn{5}{|c|}{$\begin{array}{l}\text { Table data are number of patients or median (range), or mean } \pm \text { standard deviation. } \\
\text { †P values are acquired by univariate analyses between HBeAg clearance and non-clearance.. } \\
\text { †† Thirty-nine patients with available genotype data were analyzed. } \\
\text { f Fifty-nine patients with available liver biopsy data were analyzed. Hepatic inflammation grade and } \\
\text { fibrosis stage were diagnosed according to the modified Knodell and Ishak scoring system } \\
\text { respectively. }\end{array}$} \\
\hline \multicolumn{5}{|c|}{$\begin{array}{l}\text { Abbrevation: ADV, Adefovir; ALP, alkaline phosphatase; ALT, alanine aminotransferase; AST, aspartate } \\
\text { aminotransferase; BMI, body mass index; ccCDNA, covalently closed circular DNA; ETV, Entecavir; HBV, } \\
\text { hepatitis B virus; HBV RNA, hepatitis B virus ribonucleic acid; HBcrAg, hepatitis B core-related antigen; } \\
\text { HBeAg, hepatitis B e antigen; HBsAg, hepatitis B surface antigen; TBIL, total bilirubin. }\end{array}$} \\
\hline
\end{tabular}

Percentages of patients followed-up at each time point for HBeAg clearance and seroconversion were shown in Supplementary Materials and Supplementary Fig. 1.

\section{Dynamic changes of HBV viral markers during the long-term NAs therapy}

As shown in Figs. 1 \& 2, baseline serum HBV DNA, HBV RNA, HBcrAg, and HBsAg levels were $6.44 \pm 1.95$ $\log _{10} \mathrm{IU} / \mathrm{mL}, 5.38 \pm 1.71 \log _{10}$ copies $/ \mathrm{mL}, 7.15 \pm 1.12 \log _{10} \mathrm{U} / \mathrm{mL}$ and $3.60 \pm 1.04 \log _{10} \mathrm{IU} / \mathrm{mL}$, respectively. After 72 months' NAs treatment, these levels decreased to $1.70 \pm 0.00 \log _{10} \mathrm{lU} / \mathrm{mL}, 2.62 \pm 1.42$ $\log _{10}$ copies $/ \mathrm{mL}, 4.89 \pm 1.02 \log _{10} \mathrm{U} / \mathrm{mL}$ and $2.94 \pm 1.18 \log _{10} \mathrm{IU} / \mathrm{mL}$, respectively (all $P<0.05$ ). Baseline serum intrahepatic HBV DNA and cccDNA levels were $6.37 \pm 0.83 \log _{10}$ copies $/ 10^{5}$ cells and $4.74 \pm 0.93$ $\log _{10}$ copies $/ 10^{5}$ cells, respectively. At month 60 , they were significantly decreased to $4.91 \pm 0.50$ $\log _{10}$ copies $/ 10^{5}$ cells and $3.41 \pm 0.60 \log _{10}$ copies $/ 10^{5}$ cells, respectively (both $P<0.05$ ). The level of 
these markers were significantly decreased, regardless of the occurrence of HBeAg clearance or seroconversion (all $P<0.05$ ).

\section{Relevance of viral markers at baseline and during early on- treatment for $\mathrm{HBeAg}$ clearance or seroconversion}

During follow-up, 63.86\% (53 of 83) patients achieved HBeAg clearance, and 44.58\% (37/83) achieved $\mathrm{HBeAg}$ seroconversion. Univariate analysis revealed that older age, lower baseline levels of HBsAg, HBV DNA, HBV RNA, and HBcrAg, as well as higher baseline hepatic inflammation degree and fibrosis score were related to $\mathrm{HBeAg}$ clearance (all $P<0.05$ ). Further logistic regression analysis revealed that baseline HBV RNA was independently associated with $\mathrm{HBeAg}$ clearance $(\mathrm{OR}=0.50,95 \% \mathrm{Cl} 0.309-0.809, P=$ 0.005). Moreover, the serum HBV RNA level at month 6 was independently associated with $\mathrm{HBeAg}$ clearance $(\mathrm{OR}=0.42,95 \% \mathrm{Cl} 0.248-0.714, P=0.001)$, as well as the serum HBV RNA levels at month 12 (OR $=0.39,95 \% \mathrm{Cl} 0.253-0.592, P<0.001)($ Table 1$)$.

As shown in Fig. 4, the cumulative of HBeAg clearance rate increased gradually. Kaplan-Meier survival analysis showed that patients with lower baseline levels of HBV DNA (Fig. 3A), HBsAg (Fig. 3B), HBcrAg (Fig. 3C) and HBV RNA (Fig. 3D) had a higher accumulative HBeAg clearance rate than those with higher levels (all $P<0.05$ ). Patients with different levels of intrahepatic HBV DNA (Fig. 3E) and cccDNA (Fig. 3F) showed no statistical differences of accumulative HBeAg clearance rate (both $P>0.05$ ). Cox multivariate analysis showed that an HBV RNA $<5.45 \log _{10}$ copies/mL was independently associated with HBeAg clearance $(\mathrm{HR}=5.06,95 \% \mathrm{Cl} 1.87-13.71, P=0.001)$. The result of other markers were shown in Supplementary Table 1.

Regarding to the occurrence of HBeAg seroconversion, univariate analysis showed that lower baseline levels of HBV DNA, HBV RNA and higher hepatic fibrosis score were related to HBeAg seroconversion. Logistic regression analysis revealed that baseline HBV RNA was the only viral maker independently associated with $\mathrm{HBeAg}$ seroconversion (OR $=0.689,95 \% \mathrm{Cl} 0.513-0.925, P=0.013)$. Interestingly, the result revealed that in addition to the baseline, serum HBV RNA at month $6(\mathrm{OR}=0.44,95 \% \mathrm{Cl} 0.260-$ $0.744, P=0.002)$ and month $12(\mathrm{OR}=0.58,95 \% \mathrm{Cl} 0.427-0.798, P=0.001)$ remained to be independently and negatively associated with HBeAg seroconversion (Table 2). 
Table 2

The analysis of clinical variables related to HBeAg seroconversion in univariate analyses

Variables

$\begin{array}{lll}\begin{array}{l}\text { HBeAg } \\ \text { seroconversion }\end{array} & \begin{array}{l}\text { HBeAg non- } \\ \text { seroconversion } \\ (n=37)\end{array} & \begin{array}{l}P \\ \text { value }\end{array} \\ & (n=46) & \end{array}$

\section{Baseline characteristics}

\begin{tabular}{|c|c|c|c|}
\hline Age, yrs & $37.84 \pm 10.51$ & $35.28 \pm 9.12$ & 0.239 \\
\hline Male (\%) & $28(75.68)$ & $40(87.00)$ & 0.184 \\
\hline ETV/ADV & $18 / 19$ & $28 / 18$ & 0.266 \\
\hline $\mathrm{BMI} \mathrm{Kg/ \textrm {m } ^ { 2 }}$ & $24.24 \pm 3.18$ & $23.61 \pm 4.10$ & 0.446 \\
\hline HBV Genotype † & $1 / 13 / 2$ & $4 / 16 / 3$ & 0.526 \\
\hline \multicolumn{4}{|l|}{$\mathrm{B} / \mathrm{C} /$ others } \\
\hline$A L T, U / L$ & $97.63 \pm 99.98$ & $98.88 \pm 115.97$ & 0.960 \\
\hline AST, U/L & $63.60 \pm 61.40$ & $56.18 \pm 46.68$ & 0.714 \\
\hline AST/ALT ratio & $0.74 \pm 0.35$ & $0.78 \pm 0.32$ & 0.431 \\
\hline TBIL, $\mu \mathrm{mol} / \mathrm{L}$ & $16.86 \pm 7.19$ & $16.64 \pm 8.11$ & 0.752 \\
\hline ALP, U/L & $1059.51 \pm 51.55$ & $104.60 \pm 66.52$ & 0.819 \\
\hline $\mathrm{HBsAg}\left(\log _{10} \mathrm{IU} / \mathrm{mL}\right)$ & $3.51 \pm 0.71$ & $3.66 \pm 1.24$ & 0.098 \\
\hline HBV DNA $\left(\log _{10} \mathrm{IU} / \mathrm{mL}\right)$ & $5.92 \pm 2.00$ & $6.87 \pm 1.83$ & 0.019 \\
\hline HBV RNA ( $\log _{10}$ copies/mL ) & $4.80 \pm 1.64$ & $5.82 \pm 1.65$ & 0.010 \\
\hline $\mathrm{HBcrAg}\left(\log _{10} \mathrm{U} / \mathrm{mL}\right)$ & $6.75 \pm 1.25$ & $7.71 \pm 0.96$ & 0.054 \\
\hline $\begin{array}{l}\text { Intrahepatic HBV DNA ( } \log _{10} \text { copies } / 10^{5} \\
\text { cell) }\end{array}$ & $6.27 \pm 0.77$ & $6.45 \pm 0.88$ & 0.454 \\
\hline $\operatorname{cccDNA}\left(\log _{10}\right.$ copies $/ 10^{5}$ cell) & $4.54 \pm 0.82$ & $4.90 \pm 0.99$ & 0.160 \\
\hline
\end{tabular}

† Thirty-nine patients with available genotype data were analyzed

‡ Fifty-nine patients with available liver biopsy data were analyzed. Hepatic inflammation grade and fibrosis stage were diagnosed according to the modified knodell and Ishak scoring system respectively.

Abbrevation: ADV, Adefovir; ALP, alkaline phosphatase; ALT, alanine aminotransferase; AST, aspartate aminotransferase; BMI, body mass index; cccDNA, covalently closed circular DNA; ETV, Entecavir; HBV, hepatitis B virus; HBV RNA, hepatitis B virus ribonucleic acid; HBcrAg, hepatitis B core-related antigen; HBeAg, hepatitis B e antigen; HBsAg, hepatitis B surface antigen; TBIL, total bilirubin. 


\begin{tabular}{|c|c|c|c|}
\hline Variables & $\begin{array}{l}\mathrm{HBeAg} \\
\text { seroconversion } \\
(\mathrm{n}=37)\end{array}$ & $\begin{array}{l}\text { HBeAg non- } \\
\text { seroconversion } \\
(n=46)\end{array}$ & $\begin{array}{l}P \\
\text { value }\end{array}$ \\
\hline Inflammation scores $\ddagger$ & $9.65 \pm 3.99$ & $7.74 \pm 3.56$ & 0.056 \\
\hline Fibrosis scoresł & $3.33 \pm 0.95$ & $2.86 \pm 0.91$ & 0.040 \\
\hline \multicolumn{4}{|l|}{ At 6 month time-point } \\
\hline Age, yrs & $37.84 \pm 10.51$ & $35.28 \pm 9.12$ & 0.239 \\
\hline$A L T, U / L$ & $34.36 \pm 21.63$ & $42.69 \pm 36.33$ & 0.694 \\
\hline AST, U/L & $30.98 \pm 13.63$ & $31.67 \pm 15.24$ & 0.987 \\
\hline AST/ALT ratio & $1.01 \pm 0.30$ & $0.91 \pm 0.29$ & 0.265 \\
\hline TBIL, $\mu \mathrm{mol} / \mathrm{L}$ & $17.56 \pm 8.20$ & $17.21 \pm 6.64$ & 0.884 \\
\hline ALP, U/L & $103.53 \pm 35.40$ & $91.82 \pm 27.94$ & 0.340 \\
\hline $\mathrm{HBsAg}\left(\log _{10} \mathrm{IU} / \mathrm{mL}\right)$ & $3.53 \pm 0.66$ & $3.55 \pm 0.96$ & 0.576 \\
\hline HBV DNA $\left(\log _{10} I \mathrm{IU} / \mathrm{mL}\right)$ & $2.76 \pm 1.27$ & $3.38 \pm 1.38$ & 0.036 \\
\hline HBV RNA $\left(\log _{10}\right.$ copies/mL ) & $3.64 \pm 1.50$ & $5.22 \pm 1.79$ & 0.000 \\
\hline $\mathrm{HBcrAg}\left(\log _{10} \mathrm{IU} / \mathrm{mL}\right)$ & $7.01 \pm 1.01$ & $6.05 \pm 1.00$ & 0.003 \\
\hline \multicolumn{4}{|l|}{ At 12 month time-point } \\
\hline Age, yrs & $38.84 \pm 10.51$ & $36.28 \pm 9.12$ & 0.239 \\
\hline HBV DNA $\left(\log _{10} I U / m L\right)$ & $2.43 \pm 1.25$ & $3.15 \pm 1.63$ & 0.014 \\
\hline $\mathrm{ALT}, \mathrm{U} / \mathrm{L}$ & $34.30 \pm 25.65$ & $30.11 \pm 17.45$ & 0.567 \\
\hline AST, U/L & $29.94 \pm 12.16$ & $26.99 \pm 10.66$ & 0.244 \\
\hline AST/ALT ratio & $1.10 \pm 0.55$ & $1.02 \pm 0.34$ & 0.682 \\
\hline TBIL, $\mu \mathrm{mol} / \mathrm{L}$ & $16.64 \pm 7.74$ & $17.36 \pm 8.43$ & 0.823 \\
\hline
\end{tabular}

† Thirty-nine patients with available genotype data were analyzed

$\ddagger$ Fifty-nine patients with available liver biopsy data were analyzed. Hepatic inflammation grade and fibrosis stage were diagnosed according to the modified knodell and Ishak scoring system respectively.

Abbrevation: ADV, Adefovir; ALP, alkaline phosphatase; ALT, alanine aminotransferase; AST, aspartate aminotransferase; BMI, body mass index; cccDNA, covalently closed circular DNA; ETV, Entecavir; HBV, hepatitis B virus; HBV RNA, hepatitis B virus ribonucleic acid; HBcrAg, hepatitis B core-related antigen; HBeAg, hepatitis B e antigen; HBsAg, hepatitis B surface antigen; TBIL, total bilirubin. 


\begin{tabular}{|c|c|c|c|}
\hline Variables & $\begin{array}{l}\text { HBeAg } \\
\text { seroconversion } \\
(n=37)\end{array}$ & $\begin{array}{l}\text { HBeAg non- } \\
\text { seroconversion } \\
(n=46)\end{array}$ & $\begin{array}{l}P \\
\text { value }\end{array}$ \\
\hline ALP, U/L & $98.83 \pm 57.25$ & $90.27 \pm 43.89$ & 0.470 \\
\hline HBV RNA ( $\log _{10}$ copies/mL ) & $3.26 \pm 1.47$ & $4.86 \pm 1.86$ & 0.000 \\
\hline \multicolumn{4}{|c|}{$\begin{array}{l}\text { † Thirty-nine patients with available genotype data were analyzed } \\
\text { ‡ Fifty-nine patients with available liver biopsy data were analyzed. Hepatic inflammation grade and } \\
\text { fibrosis stage were diagnosed according to the modified knodell and Ishak scoring system } \\
\text { respectively. }\end{array}$} \\
\hline \multicolumn{4}{|c|}{$\begin{array}{l}\text { Abbrevation: ADV, Adefovir; ALP, alkaline phosphatase; ALT, alanine aminotransferase; AST, aspartate } \\
\text { aminotransferase; BMI, body mass index; cccDNA, covalently closed circular DNA; ETV, Entecavir; HBV, } \\
\text { hepatitis B virus; HBV RNA, hepatitis B virus ribonucleic acid; HBcrAg, hepatitis B core-related antigen; } \\
\text { HBeAg, hepatitis B e antigen; HBsAg, hepatitis B surface antigen; TBIL, total bilirubin. }\end{array}$} \\
\hline
\end{tabular}

The cumulative of HBeAg seroconversion rate was gradually increased. Kaplan-Meier survival analysis showed that patients with lower baseline level of serum HBV DNA (Fig. 4A), higher baseline level of HBsAg (Fig. 4B), and lower baseline level of HBV RNA (Fig. 4C) had a higher accumulative HBeAg seroconversion rate (all $P<0.05$ ), while patients with different levels of HBcrAg (Fig. 4D), intrahepatic HBV DNA (Fig. 4E) and cccDNA (Fig. 4F) had no statistical differences in accumulative HBeAg seroconversion rate (all $P>0.05$ ). Cox multivariate analysis showed that baseline HBV RNA $<5.45$ $\log _{10}$ copies/mL was independently associated with $\mathrm{HBeAg}$ seroconversion $(\mathrm{HR}=3.38,95 \% \mathrm{Cl} 1.279-$ 8.908, $P=0.014)$. The result of other markers were shown in Supplementary Table 2.

The decline of HBV RNA during the first 6 months of treatment was significantly higher in HBeAg clearance patients than that in non-clearance patients, however, this value was comparable between HBeAg seroconversion and non-seroconversion (all $P>0.05$ ). The declines of HBV RNA after 12 months treatment were not significantly between $\mathrm{HBeAg}$ clearance/seroconversion and non-clearance/nonseroconversion (all $P>0.05$ ) (Supplementary Materials and Supplementary Table 3).

\section{Predicting potentials of baseline and early on-treatment viral markers quantification for $\mathrm{HBeAg}$ clearance and seroconversion}

With the cut-off value of $5.43 \log _{10}$ copies/mL, the area under the ROC curve (AUC) of baseline HBV RNA for predicting $\mathrm{HBeAg}$ clearance was $0.81(95 \% \mathrm{Cl}$ : $0.70-0.89)$, higher than that of $\mathrm{HBsAg}(0.78,95 \% \mathrm{Cl}$ : $0.63-0.89$,$) , HBV DNA (0.73,95 \% \mathrm{Cl}: 0.62-0.82)$ and $\mathrm{HBcrAg}(0.78,95 \% \mathrm{Cl}: 0.63-0.89)$, but the differences were not significant (all $P>0.05$ ). The sensitivity and specificity of baseline HBV RNA for predicting HBeAg clearance were $68 \%$ (95\% Cl: $0.53-0.81)$ and $81 \%$ (95\% Cl: $0.62-0.94)$, respectively (Supplementary Table 4). 
Similarly, with the cut-off value of $5.78 \log _{10}$ copies $/ \mathrm{mL}$, the AUC of baseline HBV RNA for predicting HBeAg seroconversion was 0.68 (95\% Cl: $0.56-0.78)$, higher than that of $\mathrm{HBsAg}(0.64,95 \% \mathrm{Cl}: 0.49-0.80)$, HBV DNA $(0.65,95 \% \mathrm{Cl}: 0.54-0.75)$ and $\mathrm{HBcrAg}(0.65,95 \% \mathrm{Cl}: 0.49-0.78)$. Again, the differences were not significantly (all $P>0.05$ ). The sensitivity and specificity of baseline HBV RNA for predicting HBeAg seroconversion were $72 \%$ (95\% Cl: $0.53-0.86$ ) and 57\% (95\% Cl: $0.41-0.72)$, respectively.

Next, we evaluated various combination of these baseline viral biomarker to predict the HBeAg clearance and seroconversion. (Supplementary Table 4, and Supplementary Table 5). Moreover, HBV RNA levels at month 6 and month 12 on-treatment time points remained its good predictive performance for $\mathrm{HBeAg}$ clearance and seroconversion. (Supplementary Table 6, and Supplementary Table 7).

\section{Discussion}

To our knowledge, the present study was first head-to-head comparison of these serum markers quantitation in predicting the $\mathrm{HBeAg}$ response, and found that not only at baseline, but also at early-on treatment of month 6 and 12, serum HBV RNA levels were independently and negatively associated with $\mathrm{HBeAg}$ clearance and seroconversion. Besides, the AUC of HBV RNA levels for HBeAg clearance and seroconversion was the highest among these viral markers.

According to our results, HBeAg clearance patients have significant lower baseline HBsAg, HBV DNA, HBV RNA, and HBcrAg than those non-clearance patients. However, HBeAg seroconversion patients did not show significant lower baseline HBsAg and HBcrAg than those non-seroconversion. The result of $\mathrm{HBsAg}$ in patients with $\mathrm{HBeAg}$ seroconversion seems consistent with previous study that patients with $\mathrm{HBeAg}$ seroconversion have lower baseline HBsAg than these patients without seroconversion despite the difference was not significant $[9,11,23]$, we also noticed that patients with higher level of baseline HBsAg showed a higher accumulative HBeAg seroconversion rate, the discrepancy maybe due to the relative small sample size of patients. Our result of HBcrAg was in line with Wang's study that HBcrAg baseline level of patients who achieved HBeAg seroconversion was not significantly lower than patients with nonseroconversion [19].

The predictive performance of HBV RNA for HBeAg clearance and seroconversion was better than that of HBsAg, HBV DNA or HBcrAg in our study, the underlying mechanism may be as follows: Firstly, the HBsAg is produced both from cccDNA and HBV DNA integrated into the host genome [2], negatively affected the prediction for HBeAg clearance and seroconversion. Secondly, circulating HBV DNA decline rapidly after NAs treatment, this characteristic restricted its use for the prediction of HBeAg clearance and seroconversion after long-term NAs treatment. Thirdly, the viral mutation, especially precore/core sequences, influencing the HBeAg seroconversion had been reported previously [24], which may affected the predictive performance of HBcrAg for the HBeAg clearance and seroconversion. Last but not least, anti-HBe antibodies are cross-reactive with HBcAg due to the amino acid sequence homology [25], and p22cr (also detected as a part of HBcrAg) was found in 'empty' HBV DNA-negative Dana particles [26], these factors may resulting in high interference against accurate measurement of the level of HBcrAg. All 
these factors may hinder the use of these markers for predicting HBeAg clearance and seroconversion. However, further research is needed to clarify the underlying mechanism.

Rapid decrease of HBV RNA in the early NAs treatment are likely associated with HBeAg clearance and seroconversion [23]. Whether or not the differences were significant, our result showed that patients who achieved HBeAg clearance or seroconversion have higher HBV RNA decline after 6 or 12 months treatment than those with non-clearance or non-seroconversion, which seems consistent with Wang's study [23]. Moreover, we also found that the AUC of different combination models based on HBV RNA, HBsAg, HBV DNA, or HBcrAg only slightly increase compared with HBV RNA alone and the difference were not statistically significant. However, in a previous study [19], a combination of HBsAg and HBcrAg had the greatest predictive value for HBeAg seroconversion, with AUC of 0.769 at month $6,0.807$ at month 12. The discrepancy maybe due to the difference of combined method of variable, the genotype make-up of patients, and HBV RNA was not included in that study. Thirdly, from the viewpoint of HBV RNA, despite the difference were not significant, the AUC tended to increase from 0.81 and 0.68 at baseline to 0.86 and 0.75 at month $6,0.86$ and 0.74 at month 12 for the prediction of HBeAg clearance and seroconversion, which still consistent with Wang's study [23]. Due to the deficiency of HBsAg and HBcrAg quantification at month 12, whether serum HBV RNA is still superior to HBsAg and HBcrAg at month 12 for the prediction of HBeAg response warrants additional study.

Our study has limitations. Firstly, the research is single center, and the sample size is relatively small. Secondly, the increased number of lost follow-up patients after 96 months might underestimate the accumulative HBeAg clearance or seroconversion rate. Future study with larger sample size is needed to confirm our conclusion.

In conclusion, the predictive performance of HBV RNA at baseline and at month 6 post-NAs treatment for $\mathrm{HBeAg}$ clearance and seroconversion was better than that of HBsAg, HBV DNA and HBcrAg. This result deepens our knowledge and understanding of clinical significance of HBV RNA in HBeAg positive patients receiving long-term NAs treatment.

\section{Abbreviations}

ADV, Adefovir; ALT, alanine aminotransferase; AST, aspartate aminotransferase; AUC, area under the receiver operating characteristic curve; $B M I$, body mass index; $\operatorname{cccDNA}$, covalently closed circular DNA; $\mathrm{CHB}$, chronic hepatitis $\mathrm{B}$; $\mathrm{COI}$, cut-off index; HBV, hepatitis B virus; $\mathrm{HBcrAg}$, hepatitis B core-related antigen; HBeAg, hepatitis B e antigen; HBsAg, hepatitis B surface antigen; HCC, hepatocellular carcinoma; LLD, lower limit of detection; NAs, nucleos(t)ide analogues; pgRNA, pregenomic RNA; ROC, receiver operating characteristic curve.

\section{Declarations}

\section{Compliance with Ethical Requirements}


The study was conducted in compliance with ethical requirements. Use of the research samples was approved by the Medical Ethics Review Committee of Beijing YouAn Hospital.

\section{Acknowledgements}

The authors thank the participants for their contribution to the research.

\section{Funding}

This study is supported in part by National Science and Technology Key Project on "Major Infectious Diseases such as HIV/AIDS, Viral Hepatitis Prevention and Treatment" (2017ZX10302201-004, 2017ZX10202203-006, 2017ZX10201201, 2017ZX10203201-005). Beijing Municipal Administration of Hospitals Clinical medicine Development of special funding support (ZYLX202125).

\section{Conflict of interest}

The authors declare that they have no conflict of interest.

\section{Ethics approval}

The study was conducted in compliance with the Declaration of Helsinki. Use of the research samples was approved by the Medical Ethics Review Committee of Beijing YouAn Hospital.

\section{Consent to participate}

All patients provided written informed consent authorizing us to access their medical records and to store the serum specimens for research purposes.

\section{Consent for publication}

All authors read and approved the final approval of manuscript to be published.

\section{Data Availability}

The data that support the findings of this study are available from the corresponding author upon reasonable request.

\section{Code availability}

Not applicable

\section{Animal Research}

Not applicable

Plant Reproducibility 
Not applicable

\section{Clinical Trials Registration}

Not applicable

\section{Author Contributions:}

Yang Wang, Hao Liao and Zhongping Deng equally contributed to visualization, methodology, formal analysis, writing and editing the manuscript. Yanna Liu, Dandan Bian, Yan Ren, Guangxin Yu, Yingying Jiang and Li Bai contributed to data curation, acquisition of data, review and editing of the manuscript. Shuang Liu, Mei Liu, Li Zhou, Yu Chen and Zhongping Duan contributed to project administration, resources, review and editing of the manuscript.

Sujun Zheng and Fengmin Lu contributed to conceptualization, funding acquisition, designed and supervised the experiments, and critical revision of the manuscript.

\section{References}

1. Polaris Observatory C. Global prevalence, treatment, and prevention of hepatitis B virus infection in 2016: a modelling study. Lancet Gastroenterol Hepatol 2018;3:383-403.

2. Lu F, Wang J, Chen $X, X u D, X i a$ N. Potential use of serum HBV RNA in antiviral therapy for chronic hepatitis B in the era of nucleos(t)ide analogs. Front Med 2017;11:502-508.

3. Liao H, Liu Y, Li X, Wang J, Chen X, Zou J, et al. Monitoring of serum HBV RNA, HBcrAg, HBsAg and anti-HBc levels in patients during long-term nucleoside/nucleotide analogue therapy. Antivir Ther 2019;24:105-115.

4. Wang J, Du M, Huang H, Chen R, Niu J, Jiang J, et al. Reply to: "Serum HBV pgRNA as a clinical marker for cccDNA activity": Consistent loss of serum HBV RNA might predict the "para-functional cure" of chronic hepatitis B. J Hepatol 2017;66:462-463.

5. Gish RG, Chang TT, Lai CL, de Man RA, Gadano A, Llamoso C, et al. Quantitative hepatitis B surface antigen analysis in hepatitis $B$ e antigen-positive nucleoside-naive patients treated with entecavir. Antivir Ther 2013;18:691-698.

6. Zoulim F, Carosi G, Greenbloom S, Mazur W, Nguyen T, Jeffers L, et al. Quantification of HBsAg in nucleos $(\mathrm{t})$ ide-naive patients treated for chronic hepatitis $B$ with entecavir with or without tenofovir in the BE-LOW study. J Hepatol 2015;62:56-63.

7. Huang YJ, Chang CS, Peng YC, Yeh HZ, Yang SS. On-treatment HBV DNA level could predict HBeAg seroclearance in patients with HBeAg-positive chronic hepatitis B with entecavir therapy. $J$ Chin Med Assoc 2017;80:341-346.

8. Peng CY, Hsieh TC, Hsieh TY, Tseng KC, Lin CL, Su TH, et al. HBV-DNA level at 6 months of entecavir treatment predicts HBeAg loss in HBeAg-positive chronic hepatitis B patients. J Formos Med Assoc 2015;114:308-313. 
9. Lee MH, Lee DM, Kim SS, Cheong JY, Cho SW. Correlation of serum hepatitis B surface antigen level with response to entecavir in naive patients with chronic hepatitis B. J Med Virol 2011;83:1178-1186.

10. Fung J, Lai CL, Young J, Wong DK, Yuen J, Seto WK, et al. Quantitative hepatitis B surface antigen levels in patients with chronic hepatitis B after 2 years of entecavir treatment. Am J Gastroenterol 2011;106:1766-1773.

11. Shin JW, Jung SW, Park BR, Kim CJ, Eum JB, Kim BG, et al. Prediction of response to entecavir therapy in patients with HBeAg-positive chronic hepatitis B based on on-treatment $\mathrm{HBsAg}, \mathrm{HBeAg}$ and HBV DNA levels. J Viral Hepat 2012;19:724-731.

12. Wang J, Shen T, Huang X, Kumar GR, Chen X, Zeng Z, et al. Serum hepatitis B virus RNA is encapsidated pregenome RNA that may be associated with persistence of viral infection and rebound. J Hepatol 2016;65:700-710.

13. Wang J, Yu Y, Li G, Shen C, Meng Z, Zheng J, et al. Relationship between serum HBV-RNA levels and intrahepatic viral as well as histologic activity markers in entecavir-treated patients. J Hepatol 2017;S0168-8278(17):32261-32264.

14. Luo H, Zhang XX, Cao LH, Tan N, Kang Q, Xi HL, et al. Serum hepatitis B virus RNA is a predictor of HBeAg seroconversion and virological response with entecavir treatment in chronic hepatitis $B$ patients. World J Gastroenterol 2019;25:719-728.

15. Luo H, Tan N, Kang Q, Pan J, Chen H, Xi H, et al. Hepatitis B virus pregenomic RNA status can reveal the long-term prognoses of chronic hepatitis $B$ patients treated with nucleos(t)ide analogues. J Viral Hepat 2020;27:323-328.

16. Mak LY, Wong DK, Cheung KS, Seto WK, Lai CL, Yuen MF. Review article: hepatitis B core-related antigen (HBcrAg): an emerging marker for chronic hepatitis B virus infection. Aliment Pharmacol Ther 2018;47:43-54.

17. Chen EQ, Wang ML, Tao YC, Wu DB, Liao J, He M, et al. Serum HBcrAg is better than HBV RNA and HBsAg in reflecting intrahepatic covalently closed circular DNA. J Viral Hepat 2019;26:586-595.

18. Wang ML, Chen EQ, Tao CM, Tang H. Letter: serum HBcrAg is a useful marker for disease monitoring, predicting treatment response and disease outcome of chronic hepatitis B virus infection. Aliment Pharmacol Ther 2018;47:1719-1720.

19. Wang B, Carey I, Bruce M, Montague S, Dusheiko G, Agarwal K. HBsAg and HBcrAg as predictors of $\mathrm{HBeAg}$ seroconversion in HBeAg-positive patients treated with nucleos(t)ide analogues. J Viral Hepat 2018;25:886-893.

20. Lok AS, McMahon BJ. Chronic hepatitis B. Hepatology 2007;45:507-539.

21. Goodman ZD. Grading and staging systems for inflammation and fibrosis in chronic liver diseases. J Hepatol 2007;47:598-607.

22. Huang H, Wang J, Li W, Chen R, Chen X, Zhang F, et al. Serum HBV DNA plus RNA shows superiority in reflecting the activity of intrahepatic cccDNA in treatment-naive HBV-infected individuals. J Clin Virol 2018;99-100:71-78. 
23. Wang X, Wang Z, Chi X, Wu R, Jin Q, Xu H, et al. Efficacy of a combination of HBV RNA and HBeAg in predicting HBeAg seroconversion in patients treated with entecavir for 144 weeks. Int $\mathrm{J}$ Infect Dis 2020;99:171-178.

24. Sonneveld MJ, Rijckborst V, Zeuzem S, Heathcote EJ, Simon K, Senturk H, et al. Presence of precore and core promoter mutants limits the probability of response to peginterferon in hepatitis $\mathrm{B} e$ antigen-positive chronic hepatitis B. Hepatology 2012;56:67-75.

25. Wang SJ, Chen ZM, Wei M, Liu JQ, Li ZL, Shi TS, et al. Specific determination of hepatitis $B$ e antigen by antibodies targeting precore unique epitope facilitates clinical diagnosis and drug evaluation against hepatitis B virus infection. Emerg Microbes Infect 2021;10:37-50.

26. Kimura T, Ohno N, Terada N, Rokuhara A, Matsumoto A, Yagi S, et al. Hepatitis B virus DNA-negative dane particles lack core protein but contain a 22-kDa precore protein without C-terminal arginine-rich domain. J Biol Chem 2005;280:21713-21719.

\section{Figures}




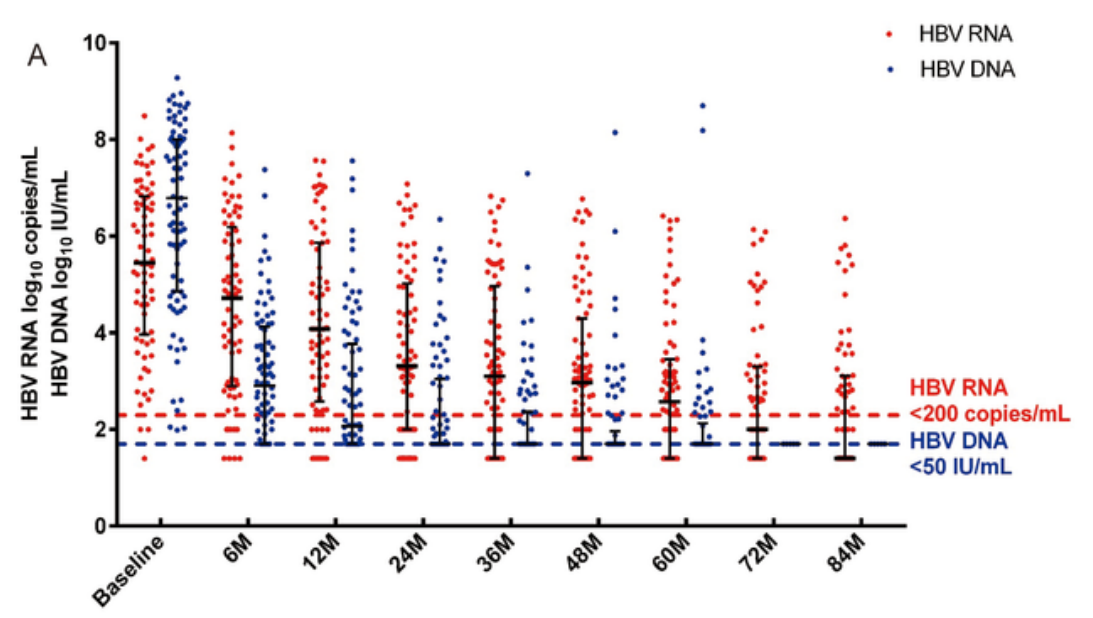

NO. of of patients being tested

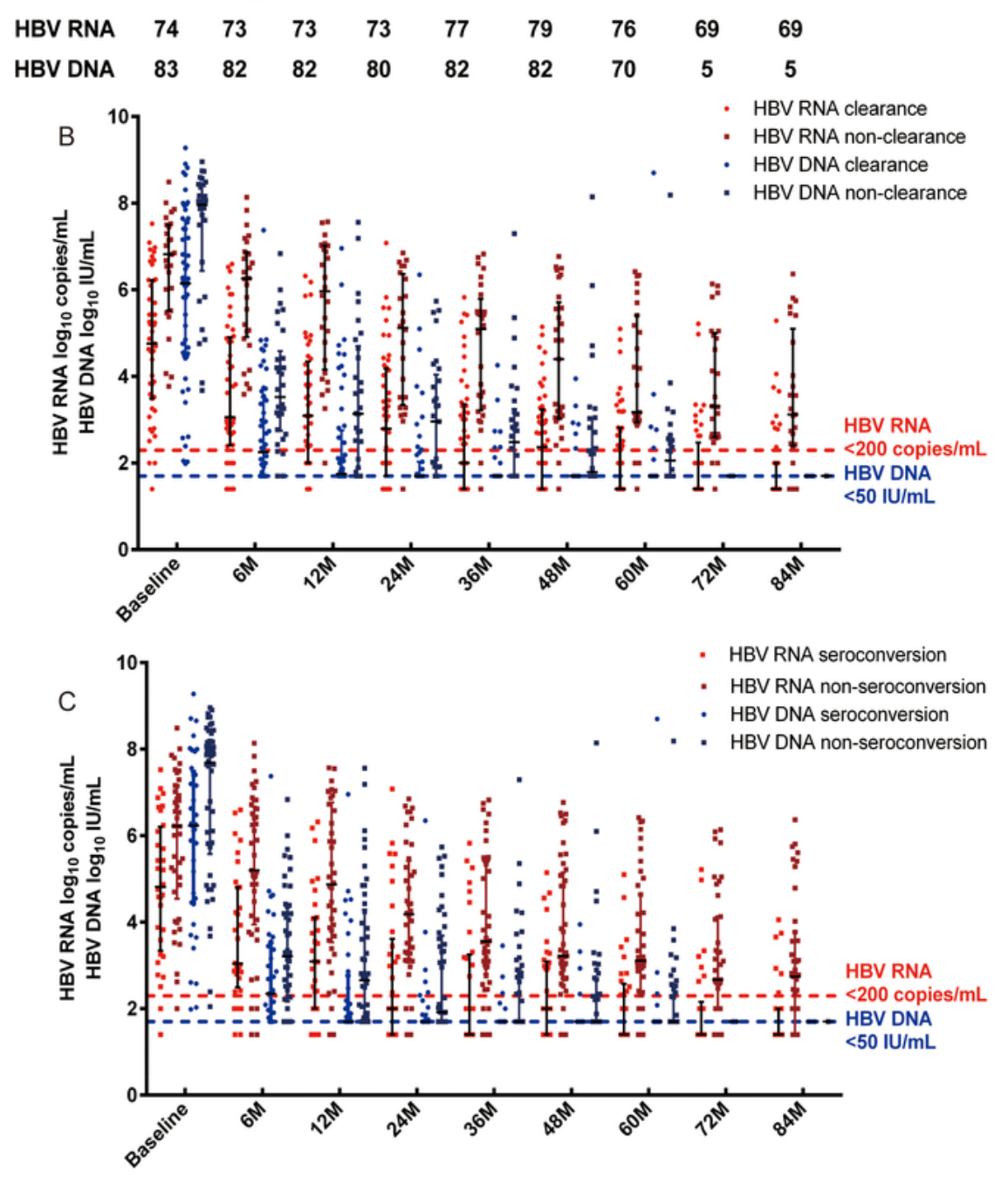

\section{Figure 1}

Dynamic changes of HBV RNA and HBV DNA levels in all patients (A), HBeAg clearance patients (B), and HBeAg seroconversion patients (C). Values lower than lower limit of detection (LLD) is designated as constant LLD 

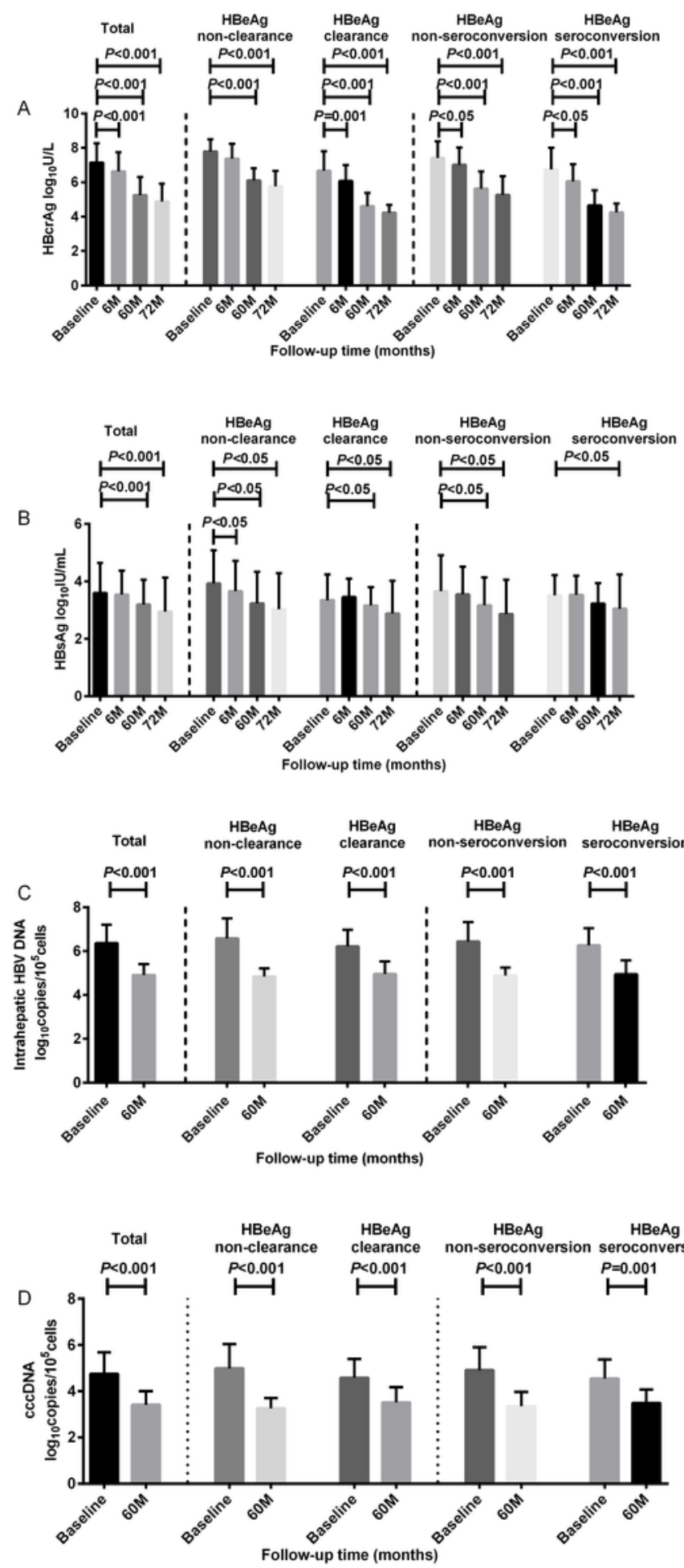

\section{Figure 2}

Dynamic changes of HBcrAg (A), HBsAg (B), intrahepatic HBV DNA (C) and cccDNA (D) in patients 

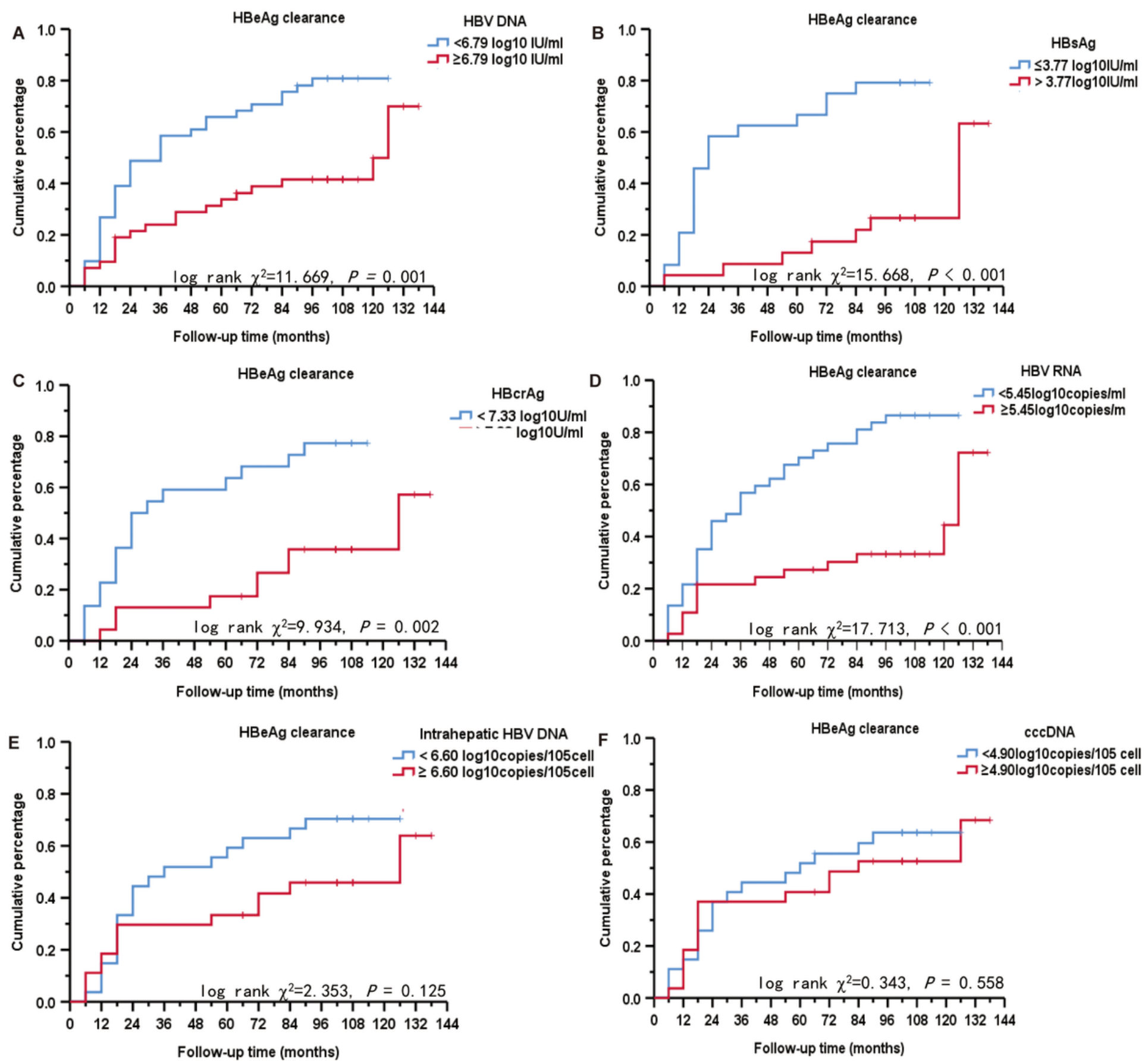

Figure 3

Kaplan-Meier survival analysis of the accumulative HBeAg clearance rate in different groups determined by HBV DNA (A), HBsAg (B), HBcrAg (C), HBV RNA (D), intrahepatic HBV DNA (E) and cccDNA (F) levels 

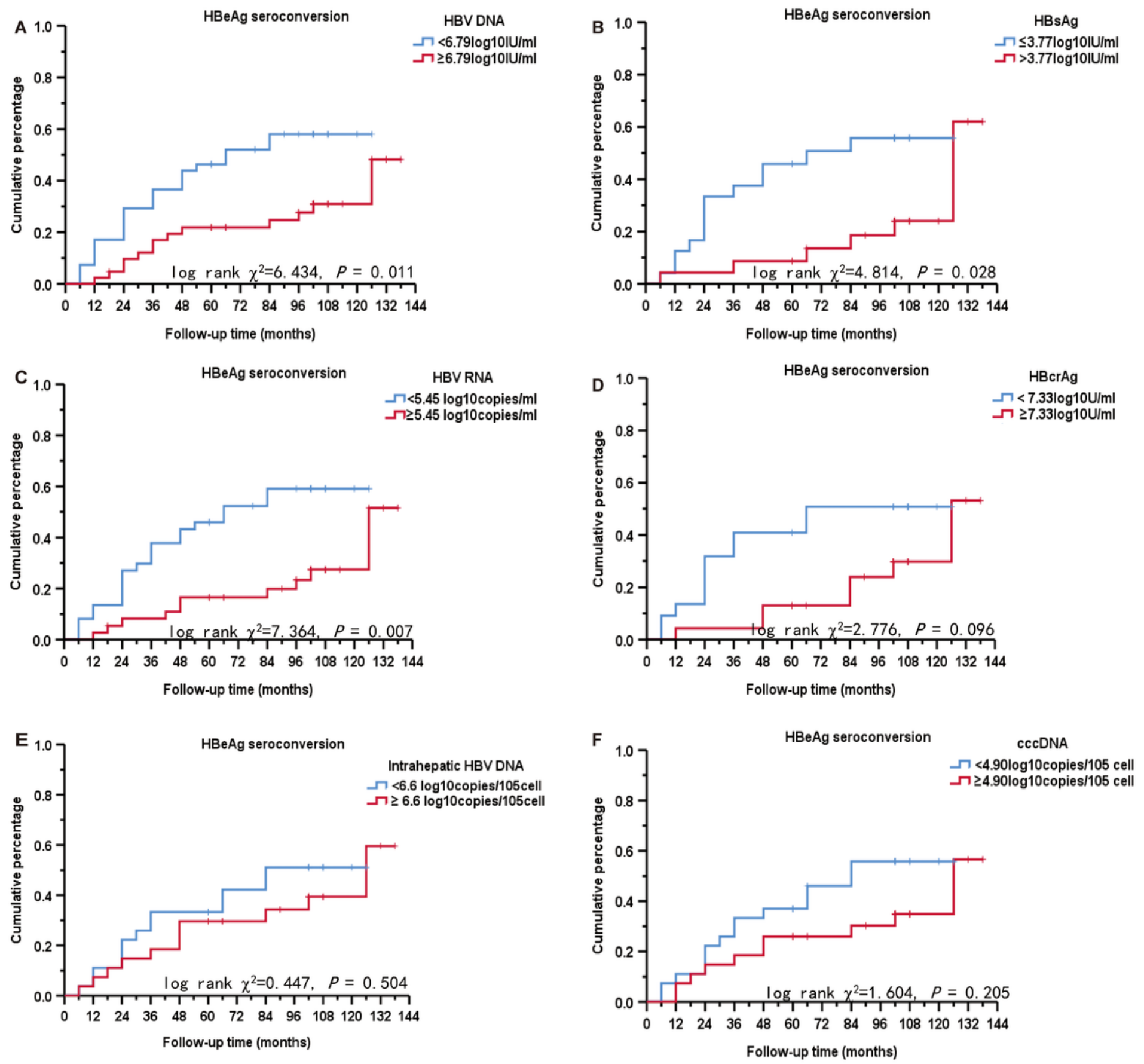

Figure 4

Kaplan-Meier survival analysis of the accumulative HBeAg seroconversion rate in different groups determined by HBV DNA (A), HBsAg (B), HBV RNA (C), HBcrAg (D), intrahepatic HBV DNA (E) and cccDNA (F) levels

\section{Supplementary Files}

This is a list of supplementary files associated with this preprint. Click to download. 
- Supplementarymaterials.docx

Page 24/24 